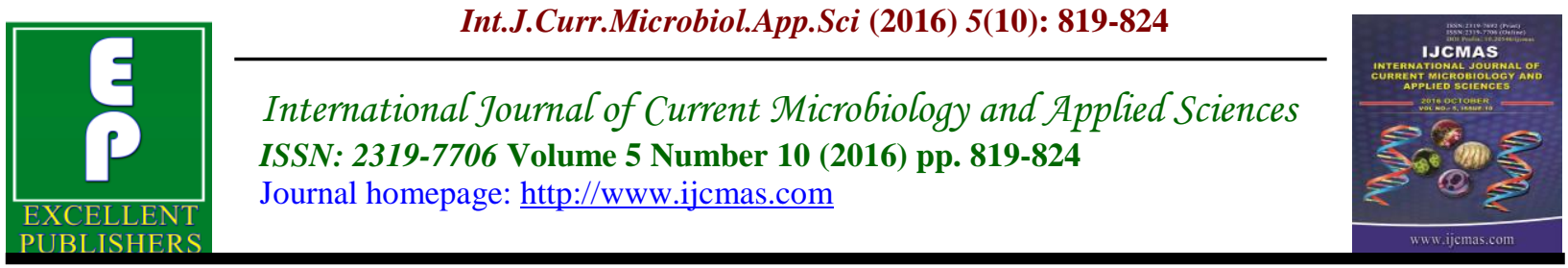

Original Research Article

http://dx.doi.org/10.20546/ijcmas.2016.510.089

\title{
Study of Seroprevalence of Dengue Virus Infection in a Tertiary Care Hospital in Patan, Gujarat, India
}

\author{
Ankita Nisarta* and Hitesh Ahir \\ Department of Microbiology, GMERS Medical College, Dharpur- Patan, Gujarat, India \\ *Corresponding author
}

\begin{abstract}
A B S T R A C T
Keywords

Dengue

diagnosis,

NS1 antigen

assay,

Dengue IgM.

Article Info

Accepted:

25 September 2016

Available Online:

10 October 2016

Dengue is a growing public health problem globally including Gujarat. This study is carried out to find out the sero-prevalence of dengue fever in Patan.There are no specific treatment or vaccine against DF or DHF. The outcome of patients with DF or DHF depends significantly on early diagnosis, leading to appropriate clinical management. This study was conducted to report the prevalence of Dengue virus infection in Patan, Gujarat. Blood samples from clinically suspected dengue cases of GMERS Medical college and hospital, Dharpur-Patan, Gujarat from 2015-2016 were collected. Samples from fever cases $<5$ days duration were tested for dengue NS1 antigen employing Pan Bio (Australia) NS1 ELISA kit. Serum samples of $\geq 5$ days fever were tested for dengue-specific IgM by MAC ELISA test kit prepared by the National Institute of Virology Pune. Out of total 90 clinically suspected dengue, 21 were laboratory-confirmed dengue cases. Among the seropositive cases $10(47.61 \%)$ were IgM positive, $17(82 \%)$ were NS1Ag positive. The majority were males $(65 \%)$ and in the age group of 16-35 years. Most of the cases were reported in monsoon and post-monsoon periods indicating increased vector transmission .A high percentage of dengue positive cases among suspected patients demands early careful investigation and management.NS1 is useful for early dengue diagnosis, although in terms of cost, ease of performance and rapidity. NS1 in combination with IgM assay offers the most sensitive and cost-effective diagnostic modality for dengue.
\end{abstract}

\section{Introduction}

Dengue fever (DF) is a self-limiting disease; however, dengue hemorrhagic fever (DHF) and dengue shock syndrome (DSS) are fatal. Since there is no specific therapy and vaccine against dengue, timely diagnosis is therefore necessary for patient management. Laboratory diagnosis is carried out by virus isolation, demonstration of viral antigen, presence of viral nucleic acid, and antibodies. Dengue is a mosquito-borne disease affecting humans mainly in tropical and subtropical regions of the world (WHO). It is an increasing public health concern in urban and suburban areas causing morbidity and mortality (Chua et al., 2011). Globally, WHO has estimated that around 3 
billion people reside in areas where there are risks of exposure to dengue virus and nearly 50 million people are infected with dengue virus every year (WHO, 2012). Dengue virus is a RNA virus, consisting of four serotypes (WHO, 2012; Chua et al., 2011; Gubler et al., 1999) all of which cause infection. Infection with one serotype does not confer cross-protection against the other serotypes, instead can cause a severe form of infection. Recently a fifth serotype was identified (Gubler et al., 1999; Gibbons et al., 2002). Early diagnosis plays a crucial role in detecting an epidemic or outbreak and in undertaking effective vector control measures.

Dengue virus infection produces a spectrum of clinical illness, ranging from an asymptomatic or mild febrile illness to classic dengue fever (DF) to the most severe form of illness, dengue hemorrhagic fever (DHF) (WHO, 2009). There is no specific treatment for dengue/ severe dengue, but early detection and access to proper medical care lowers fatality rates below 1\%.As effective control and preventive programmes for dengue infection are based upon improved surveillance data, this study was done to report the prevalence of dengue virus infection at Patan, Gujarat. It also intended to detect the epidemiological and clinical profile of dengue infection.

There are several laboratory methods available to diagnose dengue infection such as viral isolation, detection of RNA, antigen and antibody assays. However, both viral isolation and identifying viral RNA through RT-PCR are time-consuming and need a specialized laboratory with costly methods and well-trained personnel which may not be widely available in hospital settings (WHO, 2009). In most of the cases, serologic tests are used to detect dengue NS1 Ag, IgM and IgG antibodies by ELISA.
During the acute phase, the presence of IgM antibodies indicates primary infection and it appears after viremia ends or after fever subsides (Halstead et al., 2007). However, in secondary infections, IgG antibodies rise to high levels within the first week of infection and reduce over 3 to 6 months. Recently, detection of nonstructural protein 1 (NS1) antigen during the acute phase of disease in patients having primary and secondary infections has been studied in various laboratories across the world (Shrivastava et al., 2011).

\section{Materials and Methods}

The study was conducted in the Department of Microbiology, at GMERS medical college and hospital, Dharpur-patan, Gujarat. The Blood samples were collected from clinically suspected dengue fever cases. Suspected cases of dengue were defined as individuals who presented with symptoms of dengue fever and warning sign. The latter symptoms included the following: decrease in platelet count, vomiting, mucosal bleeding, lethargy, weak pulse rate, hypotension. About 2 to $3 \mathrm{ml}$ of blood was collected from each patient using strict aseptic precautions, and serum was separated by standard methods. The samples were screened for the presence of denguespecific IgM antibody capture enzyme linked immunsorbent assay(MACELISA), using a kit prepared by the National Institute of virology, Pune, India, strictly following the manufacturer's protocol (Cecilia, 2011). NS1 Ag was detected in the sera by PanBio NS1Ag ELISA kit.

\section{Statistical analysis}

Fisher's exact test was performed using GraphPad Prizm software. p-value less than 0.05 was considered significant. 


\section{Results and Discussion}

Of the total 90 clinically suspected dengue cases, 21 were laboratory-confirmed dengue cases (positive by any one of the following tests - IgM ELISA and NS1 antigen ELISA) $(23 \%)$. Out of total 90 serum samples tested, 21 were found positive for dengue virus infection. $65 \%$ positive samples were of male patients and $58 \%$ positive samples were from 16 to 35 years age group (Adult population) (58\%).

Seasonal trend showed a gradual increase in dengue positives started from August with a peak in November (34.5\%).

The most common presentation was fever $(97 \%)$ while only $1 \%$ cases presented hemorrhagic manifestations.

Most common age group affected was 0-15 years of age (32/90 - 35.55\%), followed by young adults in the age group 13-24 years $(24 / 90-26.6 \%)$.

Females $(58.5 \%)$ were affected more than the males with a male to female ratio of $1: 1.35$.

The majority of patients were from urban areas $(65 / 90,72.22 \%)$. Of these, 51 cases were from the Patan city (56.66\%).

Most of the patients in case group had fever as the presenting feature $(88 / 90,97.77 \%)$. Other clinical presentations were vomiting (64\%), headache $(37 \%)$, rash $(21 \%)$ abdominal pain (12\%) and Myalgia(7\%) Bleeding and fluid accumulation which are the signs of warning and severe dengue were seen in $22.2 \%(20 / 90)$ and $21.11 \%(19 / 90)$ of cases. (Table 2)

Of the two assays used to confirm the diagnosis of dengue, NS1 had the highest positive detection rate of $81 \%$, and $\mathrm{IgM}$ ELISA had $47.61 \%$.

Based on Fisher's exact test, the two-sided p-value was $<0.01$, considered extremely significant.

The diagnostic efficiency of NS1 antigen ELISA used in this study had $95.8 \%$ sensitivity, $\quad 54.3 \%$ specificity, $\quad 81.6 \%$ positive predictive value, $88.9 \%$ negative predictive value and 2.1 Likelihood ratio.

In this study, 23\% patients were positive for dengue infection. These findings are in accordance with other studies conducted in India by Garg (2011) and Paramasivan (2006).

The higher prevalence of dengue infection was noted among males than females. The male to female ratio was 1.9:1 which correlates well with other studies undertaken in North India and South India. High prevalence amongst males is probably due to more outdoor activities by males in comparison to females which results in more exposure to day biting mosquitoes.

Majority of infection occurred in active adults in the age group of 18-35 years. These findings are consistent with other Indian studies (Peeling et al., 2010). Active adults are doing more outdoor work so there are more chances for them to get infected. However, in studies conducted in North India (Lanciotti et al., 1992) in Chennai (Zainah et al., 2009) and in several international studies dengue has been reported to mainly a pediatric public health problem. True endemicity will be reached when the adult infection declines and only the new entrants into the population, that is, the children, are affected more by the disease. 
In the study population, highest numbers of patients were tested for dengue in the month of October followed by September and November. A gradual increase in dengue positive cases was noticed from August with a highest peak in October followed by November and September.

As analyzed from Table no.2, fever was present in almost all cases followed by myalgia, headache, joint pain, skin rash, abdominal pain and vomiting. Hemorrhagic manifestations were present in only $1 \%$ cases.

Dengue can be diagnosed by isolation of the virus, by serological tests, or by molecular methods. Diagnosis of acute (on-going) or recent dengue infection can be established by testing serum samples during the first 5 days of symptoms and/or early convalescent phase (more than 5 days of symptoms). Acute infection with dengue virus is confirmed when the virus is isolated from serum or autopsy tissue specimens, or the specific dengue virus genome is identified by reverse transcription-polymerase chain reaction (RT-PCR) from serum or plasma, cerebrospinal fluid, or autopsy tissue specimens during an acute febrile illness. Methods such as one-step, real time RTPCR or nested RT-PCR are now widely used to detect dengue viral genes in acutephase serum samples. This detection coincides with the viraemia and the febrile phase of illness onset. Acute infections can also be laboratory confirmed by identification of dengue viral antigen or RNA in autopsy tissue specimens by immunofluorescence or immunohistochemical analysis, or by seroconversion from negative to positive IgM antibody to dengue or demonstration of a fourfold or greater increase in $\mathrm{IgG}$ antibody titers in paired (acute and convalescent) serum specimens.

Table.1 Positive Result by NS1 and IgM in laboratory of confirmed dengue cases

\begin{tabular}{|l|l|}
\hline Tests & No of cases $(\mathbf{n})(\%)$ \\
\hline NS1 & $17 / 21(82 \%)$ \\
\hline Ig M & $10 / 21(47.61 \%)$ \\
\hline
\end{tabular}

Table.2 Symptoms and Complication of Confirmed Dengue cases

\begin{tabular}{|l|l|}
\hline Symptom & Percentage(\%) \\
\hline Fever & 97.77 \\
\hline vomiting & $64 \%$ \\
\hline headache & $37 \%$ \\
\hline Rash & $21 \%$ \\
\hline Abdominal pain & $12 \%$ \\
\hline Mayalgia & $7 \%$ \\
\hline $\begin{array}{l}\text { Hemorrhagic } \\
\text { Manifestation }\end{array}$ & $1 \%$ \\
\hline
\end{tabular}

NS1 antigen during the acute phase of the illness will be present in high titers in patient's sera. It can be detected both in primary and secondary infections, but detection rate is higher in acute primary than in acute secondary infections (Zainah et al., 1999; Blacksell et al., 2008). NS1 is detectable in blood from the first day up to 
day 9 to 18 after the onset of symptoms (Kumarasamy et al., 2007). In the present study, it was detectable on all 5 samples collected on day 1 , and it was detectable on day 12 in another sample. NS1 antigen levels range from 0.004 to $2 \mu \mathrm{g} / \mathrm{ml}$ in acute phase serum, to only $0.004 \mu \mathrm{g} / \mathrm{ml}$ or less in convalescent-phase serum samples. High levels of antigenaemia correlate with the development of DHF. In the present study, a total of 22 DHF/DSS cases were NS1 positive, of which 18 were DHF and 5 were DSS cases. The optical density values of NS1 antigen ELISA among these cases were very high (average OD value-2.039), which could be taken as indirect evidence of high antigenaemia.

In the present study, using the combinations of 3 assays, a total of 90 cases were confirmed as dengue out of 115 clinically suspected cases. When taken alone, NS1 antigen detection had the highest positive detection rate of $81 \%$ and $\operatorname{IgM}$ had least 47.61\%. As these 2 markers appear at 2 different phases of the study, a combination of these tests could be expected to yield the highest positive detection rate.

In conclusion, definitive diagnosis is needed to detect early infection and initiate treatment which otherwise may result in life threatening complications. Dengue affected predominantly males and active adult population. A seasonal trend was observed for dengue infections with maximum cases in post monsoon and late monsoon months which coincides with increased breeding of mosquitoes during these seasons. Therefore, vector control measures should be started before monsoon to prevent the outbreaks of dengue. This will simultaneously solve the problem of other mosquito borne diseases like malaria, chikungunya, Japanese encephalitis and filaria.

\section{References}

Alcon, S., Talarmin, A., Debruyne, M., Falconar, A., Deubel, V., Flamand, M. 2002. Enzyme-linked immunosorbent assay specific to Dengue virus type 1 nonstructural protein NS1 reveals circulation of the antigen in the blood during the acute phase of disease in patients experiencing primary or secondary infections. J. Clin. Microbiol., 40(2): 376-81.

Blacksell, S.D., Mammen, M.P., Thongpaseuth, S., Gibbons, R.V., Jarman, R.G., Jenjaroen, K., et al. 2008. Evaluation of the Panbio dengue virus nonstructural 1 antigen detection and immunoglobulin $\mathrm{M}$ antibody enzymelinked immunosorbent assays for the diagnosis of acute dengue infections in Laos. Diagn. Microbiol. Infect. Dis., 60(1): 43-49.

Chakravarti, A., R. Kumaria. 2005. "Ecoepidemiological analysis of dengue infection during an outbreak of dengue fever, India," Virol. J., vol. 2, article 32.

Chua, K.B., Mustafa, B., Abdul Wahab, A.H., Chem, Y.K., Khairul, A.H., Kumarasamy, V., et al. 2011. A comparative evaluation of dengue diagnostic tests based on single-acute serum samples for laboratory confirmation of acute dengue. Malays. J. Pathol., 33(1):13-20, $2^{\text {nd }}$ edition. Geneva: World Health Organization. (Internet). WHO (cited 2012 May 6);Availablefrom:http://www.who.int/cs $\mathrm{r} /$ resources/publications/dengue/Dengue publication/en/.

Garg, A., Garg, J., Rao, Y.K., Upadhyay, G.C., Sakhuja, S. 2011. Prevalence of dengue among clinically suspected febrile episodes at a teaching hospital in North India. J. Infect. Dis. Immunity, 3: 85. 
Gibbons, R.V., Vaughn, D.W. 2002. Dengue: an escalating problem. $B M J$, 324(7353): 1563-66.

Gubler, D.J., Meltzer, M. 1999. Impact of dengue/dengue hemorrhagic fever on the developing world. Adv. Virus Res., 53: 35-70.

Halstead, S.B. 2007. Dengue. The Lancet, 370(9599):1644-52.

Hati, A.K. 2009. "Dengue serosurveillance in Kolkata, facing an epidemic in West Bengal, India," J. Vector Borne Dis., vol. 46, no. 3, pp. 197-204.

Kumarasamy, V., Wahab, A.H.A., Chua, S.K., Hassan, Z., Chem, Y.K., Mohamad, M., et al. 2007. Evaluation of a commercial dengue NS1 antigencapture ELISA for laboratory diagnosis of acute dengue virus infection. J. Virol. Methods, 140(1-2): 75- 79.

Lanciotti, R.S., Calisher, C.H., Gubler, D.J., Chang, G.J., Vorndam, A.V. 1992. Rapid detection and typing of dengue viruses from clinical samples by using reverse transcriptase-polymerase chain reaction. J. Clin. Microbiol., 30(3): 545-51.

Lee, J., Kim, H.Y., Chong, C.K., Song, H.O. 2015. Development and clinical evaluation of a highly accurate dengue NS1 rapid test: from the preparation of a soluble NS1 antigen to the construction of an RDT. Diagn. Microbiol. Infect. Dis., 82(2): 128- 34. doi: 10.1016/j.diagmicrobio.2015.03.009.

Epub 2015 Mar 18.

Paramasivan, R., Thenmozhi, V., Hiriyan, J., Dhananjeyan, K.J., Tyagi, B.K., Dash, A.P. 2006. Serological \& entomological investigations of an outbreak of dengue fever in certain rural areas of Kanyakumari district, Tamil Nadu. Indian J. Med. Res., 123: 697-70.

Peeling, R.W., Artsob, H., Pelegrino, J.L., Buchy, P., Cardosa, M.J., Devi, S., et al. Evaluation of diagnostic tests: dengue. Nature Rev. Microbiol., 8: S30-37.

Sarkar, J.K., S.N. Chatterjee, and S.K. Chakravarty. 1967. "Three-year study of mosquito-borne haemorrhagic fever in Calcutta," Transactions of the Royal Society of Tropical Medicine and Hygiene, vol. 61, no. 5, pp. 725-735.

Shrivastava, A., Dash, P.K., Tripathi, N.K., Sahni, A.K., Gopalan, N., Lakshmana Rao, P.V. 2011. Evaluation of a commercial Dengue NS1 enzymelinked immunosorbent assay for early diagnosis of dengue infection. Indian J. Med. Microbiol., 29(1): 51-55.

Tang, K.F., Ooi, E.E. 2012. Diagnosis of dengue: an update. Expert Rev. Anti. Infect. Ther., 10(8): 895-907.

WHO. 2009. Dengue Guidelines for diagnosis, treatment, prevention and control.

WHO. Dengue hemorrhagic fever: diagnosis, treatment, prevention and control.

Zainah, S., Wahab, A.H.A., Mariam, M., Fauziah, M.K., Khairul, A.H., Roslina, I., et al. 2009. Performance of a commercial rapid dengue NS1 antigen immunochromatography test with reference to dengue NS1 antigencapture ELISA. J. Virol. Methods, 155(2): 157-60.

\section{How to cite this article:}

Ankita Nisarta and Hitesh Ahir. 2016. Study of Seroprevalence of Dengue Virus Infection in a Tertiary Care Hospital in Patan, Gujarat, India. Int.J.Curr.Microbiol.App.Sci. 5(10): 819-824. doi: http://dx.doi.org/10.20546/ijcmas.2016.510.089 